\title{
Dyclonine and alverine citrate enhance the cytotoxic effects of proteasome inhibitor MG132 on breast cancer cells
}

\author{
DONGHONG JU, XIAOGANG WANG and YOUMING XIE \\ Barbara Ann Karmanos Cancer Institute and Department of Pathology, \\ Wayne State University School of Medicine, Detroit, MI 48201, USA
}

Received October 29, 2008; Accepted December 1, 2008

DOI: 10.3892/ijmm_00000118

\begin{abstract}
Proteasome is an important target in cancer therapy. To enhance the efficacy of proteasome inhibitors is a challenging task due to the paucity of understanding the functional interactions between proteasome and other cellular pathways in mammalian cells. Taking advantage of the knowledge gained from Saccharomyces cerevisiae, we show that dyclonine and alverine citrate, the major components of two over-the-counter medicines, can substantially enhance the cytotoxic effects of proteasome inhibitor MG132 on breast cancer cells. This study also highlights an important yeast genetic approach to identification of potential therapeutics that can be used for combination therapy with proteasome inhibitors.
\end{abstract}

\section{Introduction}

Protein degradation by the proteasome is one of the major regulatory mechanisms in the cell (1). Abnormal proteasome activity is implicated in the pathogenesis of cancers and other human diseases (2). The observations that inhibition of the proteasome activity induced apoptosis preferentially in transformed cells led to the introduction of proteasome inhibitors in clinical trials for cancer therapy (3 and refs. therein). In spite of the positive results of Bortezomib (also named Velcade or PS-341), a peptide boronate proteasome inhibitor, in treating relapsed and refractory multiple myeloma and non-Hodgkin's lymphoma, the efficacy of proteasome inhibitors on solid tumors remains limited $(3,4)$. Thus, it is imperative that new approaches are developed to increase the efficacy and applicability of proteasome inhibitors in cancer therapy. One feasible approach is combination therapy that applies a second agent along with a proteasome inhibitor. The key to success of combination therapy is to target a specific pathway, which is essential for cell growth and survival when the proteasome

Correspondence to: Dr Youming Xie, Barbara Ann Karmanos Cancer Institute, Wayne State University School of Medicine, 110 Warren Avenue, Detroit, MI 48201, USA

E-mail: xiey@karmanos.org

Key words: proteasome, proteasome inhibitor, combined therapy, dyclonine, alverine citrate, yeast genetics activity is inhibited. Although it is difficult to define such pathways in mammalian cells, recent studies in Saccharomyces cerevisiae have provided the much anticipated information.

Proteasome is highly conserved, both structurally and functionally, from yeast to humans (5). In S. cerevisiae, the proteasome genes are coordinately activated by the transcription factor Rpn4 (6-9). Deletion of RPN4 substantially decreases the expression of the proteasome genes, resulting in lower proteasome activity, which mimics the effect of proteasome inhibitors (7-9). Genome-wide analyses demonstrated that deletion of RPN4 exhibits synergistic growth defects with mutations in numerous genes involved in a variety of pathways $(10,11)$. Specifically, yeast cells bearing mutations of RPN4 and one of these genes are either inviable or grow much slower than the mutants that carry a single mutation. Most of these yeast genes have homologs in human cells. We reasoned that simultaneous targeting of the proteasome and one of the human homologs may display a synergistic effect against cancer cells.

As proof of concept in this study, we chose the ERG24 gene, which encodes the $\mathrm{C}-14$ sterol reductase essential for ergosterol biosynthesis in yeast (12). Deletion of ERG24 showed synthetic growth defects when combined with deletion of RPN4 $(10,11)$. The pathways of sterol biosynthesis in eukaryotic cells are well defined. There are two Erg24 homo$\log \mathrm{s}$ in human cells with $\mathrm{C}-14$ sterol reductase activity, including 3ß-hydroxysterol C-14 reductase and Lamin B receptor $(13,14)$. Moreover, a recent study profiling the chemical-genomic interactions in yeast identified three small molecules that can inhibit the Erg24 activity (15). These three compounds share a nitrogen-containing chemical core structure and likely target the lipid biosynthesis pathway. One of the compounds is fenpropimorph, a member of the morpholines used as antifungal agents in agriculture. The other two are dyclonine and alverine citrate, the major components of two over-the-counter medicines. Dyclonine is an oral anaesthetic found in throat lozenges, whereas alverine citrate is a commonly used smooth muscle relaxant for the treatment of irritable bowel syndrome. We decided to test if these three compounds could enhance the cytotoxic effects of proteasome inhibitor MG132 on breast cancer cells.

\section{Materials and methods}

Cell viability assay. Cell viability was measured by MTT assay as previously described (18). Cells were inoculated in 96-well plates at 4,000-6,000 cells per well $24 \mathrm{~h}$ prior to the 
A

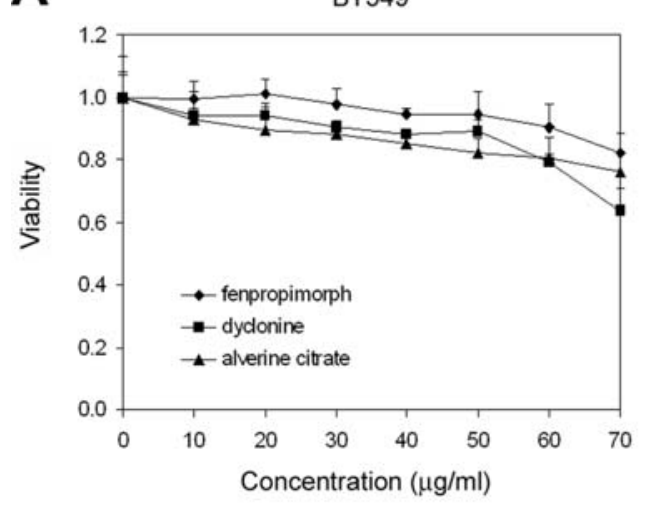

C

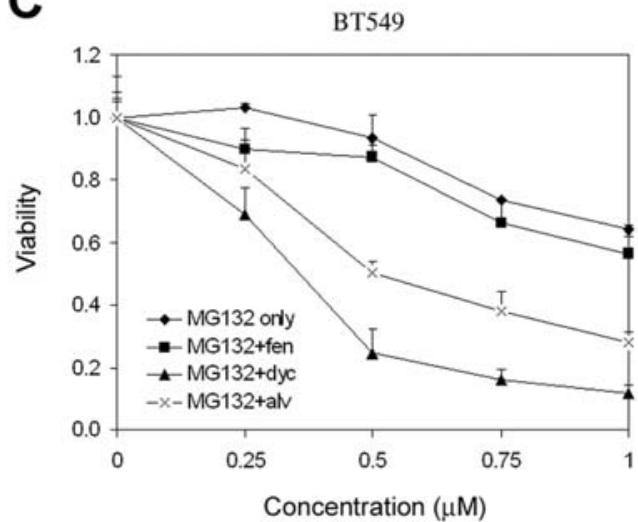

B

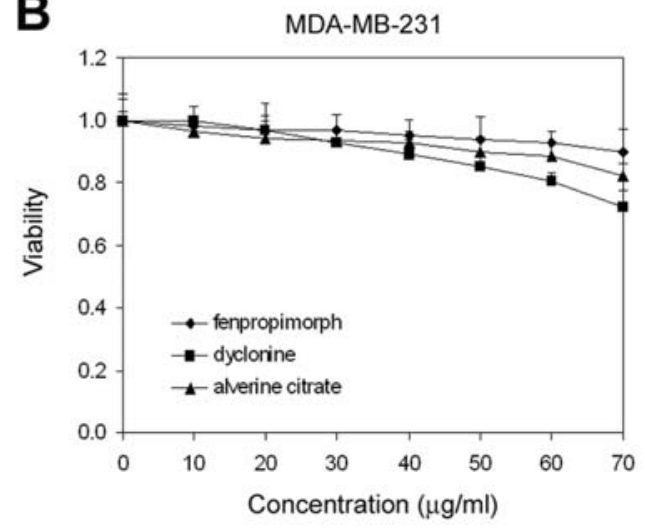

D

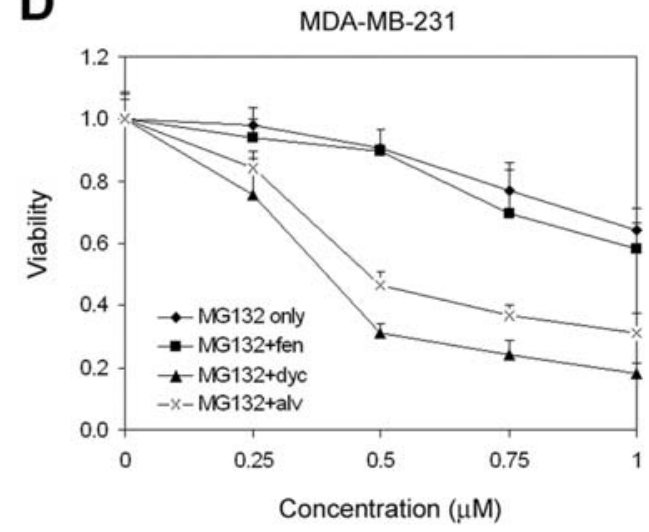

Figure 1. Dyclonine and alverine citrate enhance the cytotoxic effects of MG132. BT549 (A) and MDA-MB-231 (B) cells were treated with different concentrations of fenpropimorph, dyclonine or alverine citrate for $48 \mathrm{~h}$, and subjected to MTT assay. (C and D) Dyclonine and alverine citrate increased the cytotoxic effects of MG132. BT549 (C) and MDA-MB-231 (D) cells were treated with MG132 alone or in combination with fenpropimorph, dyclonine or alverine citrate for $48 \mathrm{~h}$. Cell viability was measured by MTT assay.

treatment with MG132, fenpropimorph, dyclonine or alverine citrate individually or combined. 3-(4,5-dimethyl-2-thiazolyl)2,5-diphenyl-2H-tetrazolium bromide (MTT), fenpropimorph, dyclonine and alverine citrate were purchased from SigmaAldrich (St. Louis, MO), whereas MG132 was obtained from BostonBiochem (Cambridge, MA). The values given are the means from three to six independent experiments.

Flow cytometry of apoptotic cells. Apoptosis was measured using Annexin V FITC Apoptosis Detection Kit according to the manufacturer's instruction (Calbiochem, San Diego, CA). After $48 \mathrm{~h}$ exposure to the drugs, cells were incubated with Annexin V-FITC and propidium iodide. The samples were analyzed by a FACScalibur flow cytometer (BectonDickinson). Intact cells are negative for both Annexin V and propidium iodide, early apoptotic cells are FITC positive but propidium iodide negative, and late apoptotic or necrotic cells are both Annexin V and propidium iodide positive. Normal cell death is recorded positive for propidium iodide only.

Immunoblot analysis. Cells were washed three times with ice-cold PBS and scraped for centrifugation. Cell pellets were lysed in buffer (50 mM Tris-Cl, $\mathrm{pH} \mathrm{7.5,} 10 \mathrm{mM} \mathrm{MgCl}_{2}$, $100 \mathrm{mM} \mathrm{KCl}, 1 \%$ Triton X-100, 10\% glycerol, $1 \mathrm{mM}$ DTT, $1 \mathrm{X}$ protease inhibitor cocktail). Cell extracts were separated by SDS-PAGE ( $8 \%$ gel), followed by immunoblotting with antiPARP (Cell Signaling, Danvers, MA) and anti- $\beta$-actin
(Sigma-Aldrich) antibodies, respectively. The signals were detected by horseradish peroxidase-conjugated secondary antibody and Visualizer Western Blot Detection Kit (Upstate, Lake Placid, NY).

\section{Results and discussion}

We first examined the possible cytotoxic effects of fenpropimorph, dyclonine and alverine citrate on breast cancer cell lines BT549 and MDA-MB-231 using MTT assay. As shown in Fig. 1A and B, these three compounds appeared to have only a mild effect at the concentrations tested. We then treated the cells with various doses of MG132 with or without addition of $30 \mu \mathrm{g} / \mathrm{ml}$ of fenpropimorph, dyclonine or alverine citrate. Remarkably, dyclonine and alverine citrate substantially enhanced the cytotoxic effects of MG132 on both cancer cell lines (Fig. 1C and D). Fenpropimorph, which has a stronger inhibitory effect on Erg24 than dyclonine and alverine citrate in yeast (15), did not increase the cytotoxic effects of MG132 (Fig. 1C and D). It is likely that the human Erg24 homologs may have a higher affinity for dyclonine and alverine citrate than fenpropimorph. In contrast, fenpropimorph may bind yeast Egr24 more efficiently than dyclonine and alverine citrate. Consistent with the MTT assay, examination of the morphological changes revealed cell death after simultaneous treatment with MG132 and dyclonine or alverine citrate (Fig. 2). By contrast, the treatment with individual agents did 


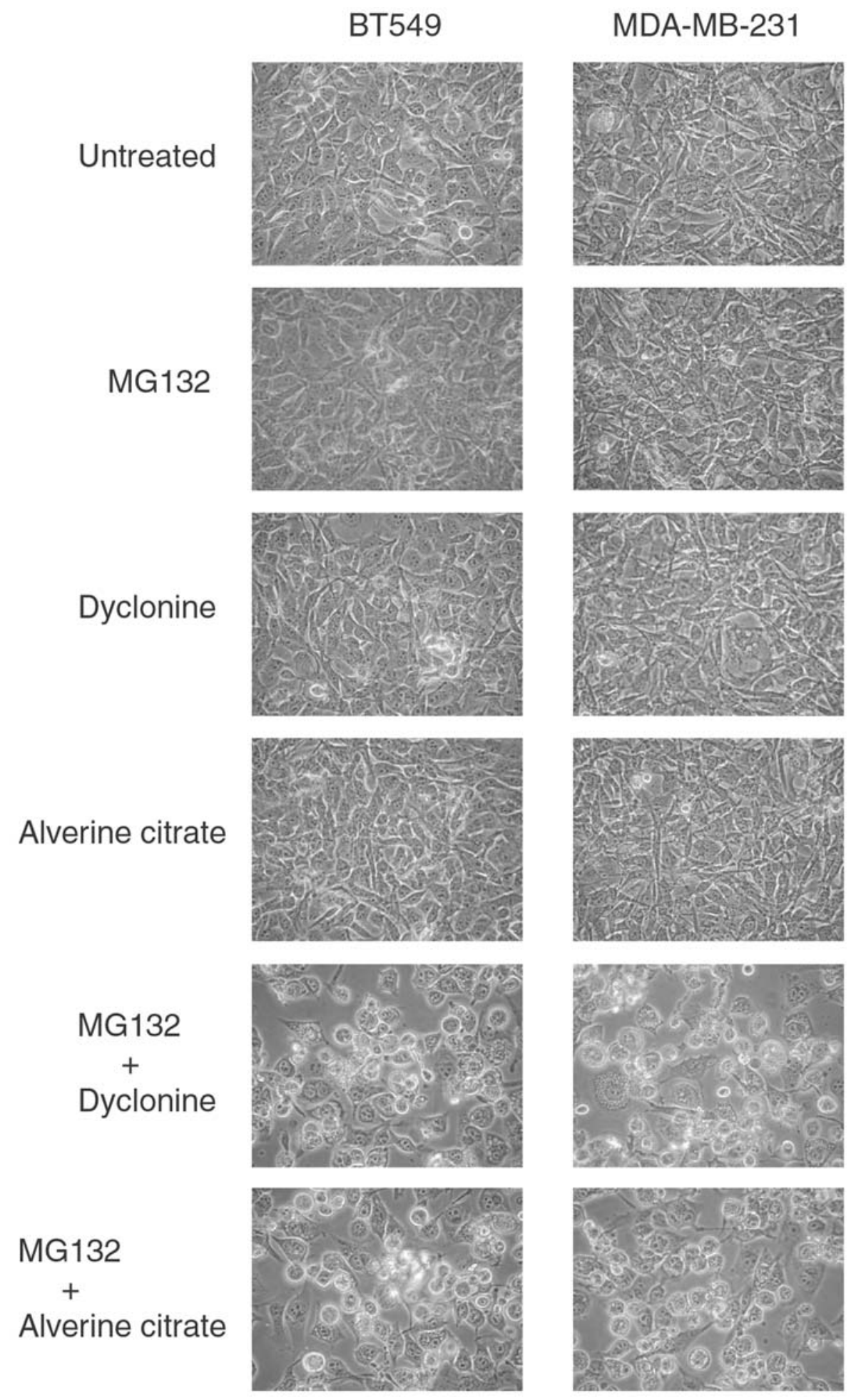

Figure 2. Combined treatment with MG132 and dyclonine or alverine citrate causes cell death. BT549 and MDA-MB-231 cells were untreated or treated with MG132 $(0.375 \mu \mathrm{M})$, dyclonine $(30 \mu \mathrm{g} / \mathrm{ml})$ or alverine citrate $(30 \mu \mathrm{g} / \mathrm{ml})$, or a combination of MG132 with dyclonine or alverine citrate for $48 \mathrm{~h}$. Morphological changes were examined by microscopy.

not cause cell death. Thus, dyclonine and alverine citrate can enhance the cytotoxic effects of MG132 on breast cancer cells.

To examine possible induction of apoptosis by the combined treatment with MG132 and dyclonine or alverine citrate, we measured the binding of fluorescein isothiocyanate (FITC)conjugated Annexin V to MDA-MB-231 cells by flow cytometry. It was demonstrated that Annexin V binds to apoptotic cells through externalized phosphatidyl serine (16). As shown in Fig. 3A, many more cells underwent apoptosis in response to the combined treatment with MG132 and dyclonine or alverine citrate than to the treatment with a single compound. Note that the combined treatment also led to a higher rate of non-apoptotic cell death, i.e. normal cell death, and that the Annexin V staining cannot definitely distinguish necrotic cells and late apoptotic cells (Fig. 3A). To further detect cell apoptosis, we applied immunoblot analysis to examine 
A
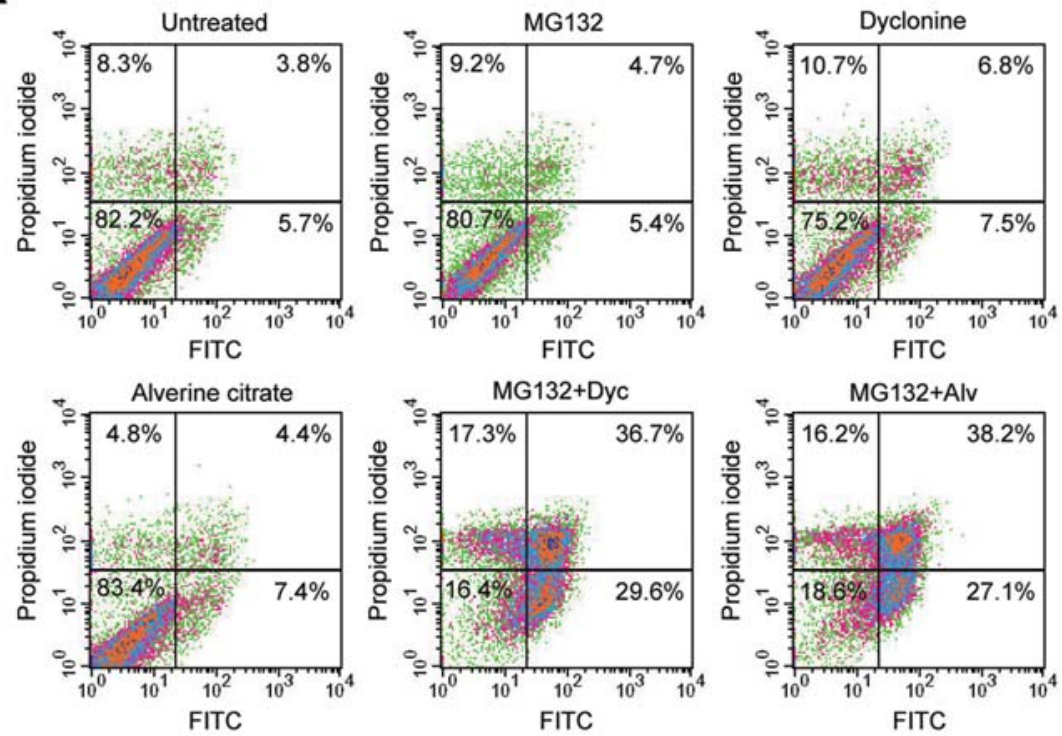

B

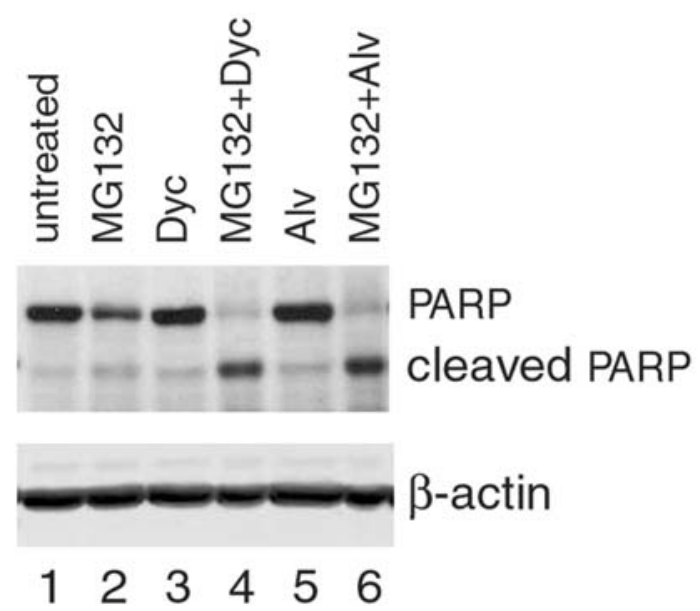

Figure 3. Apoptosis induced by a combination of MG132 with dyclonine or alverine citrate. (A) Flow cytometry of cells labeled with Annexin V-FITC and propidium iodide. MDA-MB-231 cells either untreated or treated with MG132 $(0.375 \mu \mathrm{M})$, dyclonine $(30 \mu \mathrm{g} / \mathrm{ml})$ or alverine citrate $(30 \mu \mathrm{g} / \mathrm{ml})$, or a combination of MG132 with dyclonine or alverine citrate for $48 \mathrm{~h}$ were stained with Annexin V-FITC and propidium iodide and subjected to flow cytometry. Living cells are negative for both Annexin V and propidium iodide (bottom left quadrant), early apoptotic cells are FITC positive but propidium iodide negative (bottom right quadrant), late apoptotic or necrotic cells are both Annexin V and propidium iodide positive (upper right quadrant). Normal cell death is recorded as positive for propidium iodide only (upper left quadrant). Numbers in quadrant represent the percentages of stained cells. (B) Immunoblotting analyses of PARP in BT549 cells either untreated or treated as (A). The blot was reprobed with an anti- $\beta$-actin antibody to compare the loading.

cleavage of poly (ADP-ribose) polymerase (PARP). A dramatic increase in cleaved PARP product was observed after BT549 cells were simultaneously treated with MG132 and dyclonine or alverine citrate (Fig. 3B). In contrast, the treatment with a single agent did not noticeably increase PARP cleavage. Together, these results indicate that a combination of MG132 with dyclonine or alverine citrate induces apoptosis in breast cancer cells.

The current combination therapy in clinical trials for solid tumors, including breast cancer, is virtually an application of a second anti-cancer agent along with proteasome inhibitor Bortezomib $(3,4)$. It is not surprising that such a regimen lacking a sound rationale has not produced a promising synergistic effect $(3,4)$. Taking advantage of the information gained from the budding yeast, we demonstrated that dyclonine and alverine citrate are able to enhance the cytotoxic effects of MG132 on breast cancer cells. During the course of this study, Hillenmeyer et al reported that fenpropimorph, dyclonine and alverine citrate can compromise the growth fitness of the yeast strains with heterozygous deletion of the proteasome genes (17). Thus, the functional cross-talk between downregulation of the proteasome activity and the action of dyclonine or alverine citrate is conserved in both yeast and humans.

It is unclear how exactly dyclonine and alverine citrate enhance the cytotoxic effects of MG132. Two possible mechanisms are considered. First, dyclonine and alverine citrate may facilitate MG132-induced apoptosis. It is well known that inhibition of the proteasome activity induces apoptosis in cancer cells ( 3 and refs. therein). Dyclonine and alverine citrate likely inhibit the lipid biosynthetic pathway, and, therefore, may impair the fluidity of cellular membranes, i.e. plasma and mitochondrial membranes. This may augment the induction of apoptosis by inhibition of the proteasome 
activity. Second, dyclonine and alverine citrate may have a synergistic effect with MG132 in deregulating lipid metabolism. Recent studies demonstrated that endoplasmic reticulum-associated degradation (ERAD) plays a crucial role in feedback regulation of cholesterol synthesis $(19,20)$. When sterols are depleted, the membrane-bound transcription factors (SREBPs) are activated by ERAD in which SREBPs are cleaved by the proteasome and the N-terminal fragments are released from the membrane and enter the nucleus to induce the genes required for cholesterol synthesis. On the other hand, when sterols build up in the cell, activation of SREBPs by ERAD is inhibited. We speculate that MG132 inhibits the ERAD process and, as a consequence, prevents the activation of SREBPs. Therefore, the inhibition of cholesterol synthesis by dyclonine and alverine citrate cannot be compensated by the ERAD-mediated feedback mechanism. While further investigation is needed to unveil the molecular basis of the interaction between MG132 and dyclonine/alverine citrate, our study presents a yeast genetic approach to identification of potential therapeutics that can enhance the efficacy of proteasome inhibitors. Dyclonine and alverine citrate may turn out to be two important anti-tumor agents.

\section{Acknowledgements}

We thank Eric Van Buren for his assistance in using the flow cytometry core facility of Karmanos Cancer Institute, and Dr A. Raz for BT549 and MDA-MB-231 cell lines. This work was supported by grants from the Barbara Ann Karmanos Cancer Institute and the American Cancer Society (RGS0506401-GMC).

\section{References}

1. Hershko A, Ciechanover A and Varshavsky A: The ubiquitin system. Nat Med 10: 1073-1081, 2000.

2. Schwartz AL and Ciechanover A: The ubiquitin-proteasome pathway and pathogenesis of human diseases. Annu Rev Med 50: 57-74, 1999.

3. Orlowski RZ and Kuhn DJ: Proteasome inhibitors in cancer therapy: lessons from the first decade. Clin Cancer Res 14: 1649-1657, 2008.
4. Milano A, Iaffaioli RV and Caponigro F: The proteasome: a worthwhile target for the treatment of solid tumours? Eur J Cancer 43: 1125-1133, 2007.

5. Voges D, Zwickl P and Baumeister W: The 26S proteasome: a molecular machine designed for controlled proteolysis. Annu Rev Biochem 68: 1015-1068, 1999.

6. Mannhaupt G, Schnall R, Karpov V, Vetter I and Feldmann H: Rpn4p acts as a transcription factor by binding to PACE, a nonamer box found upstream of $26 \mathrm{~S}$ proteasomal and other genes in yeast. FEBS Lett 450: 27-34, 1999.

7. Xie Y and Varshavsky A: RPN4 is a ligand, substrate, and transcriptional regulator of the $26 \mathrm{~S}$ proteasome: a negative feedback circuit. Proc Natl Acad Sci USA 98: 3056-3061, 2001.

8. Ju D, Wang L, Mao X and Xie Y: Homeostatic regulation of the proteasome via an Rpn4-dependent feedback circuit. Biochem Biophys Res Commun 321: 51-57, 2004.

9. London M, Keck BI, Ramos PC and Dohmen RJ: Regulatory mechanisms controlling biogenesis of ubiquitin and the proteasome. FEBS Lett 567: 259-264, 2004.

10. Pan X, Ye P, Yuan DS, et al: A DNA integrity network in the yeast Saccharomyces cerevisiae. Cell 124: 1069-1081, 2006.

11. Tong AH, Lesage G, Bader GD, et al: Global mapping of the yeast genetic interaction network. Science 303: 808-813, 2004.

12. Lees ND, Skaggs B, Kirsch DR and Bard M: Cloning of the late genes in the ergosterol biosynthetic pathway of Saccharomyces cerevisiae - a review. Lipids 30: 221-226, 1995.

13. Bennati AM, Castelli M, Fazia MAD, et al: Sterol dependent regulation of human TM7SF2 gene expression: role of the encoded $3 \beta$-hydroxysterol $\Delta^{14}$-reductase in human cholesterol biosynthesis. Biochim Biophys Acta 1761: 677-68, 2006.

14. Holmer L, Pezhman A and Worman HJ: The human lamin B receptor/sterol reductase multigene family. Genomics 54: 469476, 1998.

15. Giaever G, Flaherty P, Kumm J, et al: Chemogenomic profiling: identifying the functional interactions of small molecules in yeast. Proc Natl Acad Sci USA 101: 793-798, 2004.

16. Boersma AW, Nooter K, Oostrum RG and Stoter G: Quantification of apoptotic cells with fluorescein isothiocyanate-labeled annexin V in Chinese hamster ovary cell cultures treated with cisplatin. Cytometry 24: 123-130, 1996.

17. Hillenmeyer ME, Fung E, Wildenhain J, et al: The chemical genomic portrait of yeast: uncovering a phenotype for all genes. Science 320: 362-365, 2008.

18. Xu H, Ju D, Jarois T and Xie Y: Diminished feedback regulation of proteasome expression and resistance to proteasome inhibitors in breast cancer cells. Breast Cancer Res Treat 107: 267-274, 2008.

19. Meusser B, Hirsch C, Jarosch E and Sommer T: ERAD: the long road to destruction. Nat Cell Biol 7: 766-772, 2005.

20. Goldstein JL, DeBose-Boyd RA and Brown MS: Protein sensors for membrane sterols. Cell 124: 35-46, 2006. 